Article

\title{
Applications of Unmanned Aerial Systems (UAS): A Delphi Study Projecting Future UAS Missions and Relevant Challenges
}

\author{
Alberto Sigala ${ }^{1}$ and Brent Langhals ${ }^{2, *(1)}$ \\ 1 Air Force Life Cycle Management Center, Wright-Patterson AFB, OH 45433, USA; alberto.sigala@us.af.mil \\ 2 Air Force Institute of Technology, Wright-Patterson AFB, OH 45433, USA \\ * Correspondence: brent.langhals@afit.edu; Tel.: +1-937-255-3751 (ext. 7402)
}

Received: 6 February 2020; Accepted: 6 March 2020; Published: 10 March 2020

\begin{abstract}
Over recent decades, the world has experienced a growing demand for and reliance upon unmanned aerial systems (UAS) to perform a broad spectrum of applications to include military operations such as surveillance/reconnaissance and strike/attack. As UAS technology matures and capabilities expand, especially with respect to increased autonomy, acquisition professionals and operational decision makers must determine how best to incorporate advanced capabilities into existing and emerging mission areas. This research seeks to predict which autonomous UAS capabilities are most likely to emerge over the next 20 years as well as the key challenges for implementation for each capability. Employing the Delphi method and relying on subject matter experts from operations, acquisitions and academia, future autonomous UAS mission areas and the corresponding level of autonomy are forecasted. The study finds consensus for a broad range of increased UAS capabilities with ever increasing levels of autonomy, but found the most promising areas for research and development to include intelligence, surveillance, and reconnaissance (ISR) mission areas and sense and avoid and data link technologies.
\end{abstract}

Keywords: Unmanned Aerial Systems (UAS), autonomy; Delphi Study; challenges; future military applications; Data-Driven Decision-Making (DDDM)

\section{Introduction}

The history of unmanned aerial systems (UAS) is as old as the history of military aviation [1]. References to unmanned aircraft first appear with the use of hot air balloons for aerial bombardment of Venice in 1849 and again later in the American Civil War. Discussion of aerial tactics from WWI and WWII are also littered with many examples of attempts to use UAS, albeit with limited success [2]. Today, UAS are typically considered autonomous or semi-autonomous remotely piloted aircraft which mimic the maneuvers of a human-piloted aircraft without a pilot physically on board. Most UAS are currently remotely operated by pilots on the ground within a human-in-the-loop (HITL) construct or with a pilot at the controls with little autonomous functionality. However, it is believed that increased levels of autonomous UAS capabilities will develop in pace with advances in technology [3-6]. Given such expectations, future autonomous UAS uses are being discussed without a deep understanding of the implications autonomous systems present in terms of the cost, complexity, and limitations (political/legal/technical) of developing and integrating them into operational environments.

For example, over the last two decades, UAS technology has developed at a rapid pace allowing UAS to become an integral part in military operations [7]. Countries who have acquired armed UAS has grown from three countries (the US, UK, and Israel) in 2008, to at least 28 in 2018; with 10 countries 
having reportedly used them in combat [8]. Outside of military operations, the UAS sector growth is predicted to continue to expand and was described as "the most dynamic growth sector of the world aerospace industry this decade" [9].

The United States military, a leader in UAS development and use, has realized year-over-year increases in research expenditures into unmanned systems. The left side of Figure 1 depicts the increased funding for general unmanned systems by military branch, while, the right-hand side of the figure clearly indicates UAS as the largest funded portion of the unmanned systems budget. By some estimates, military research expenditure for UAS technology is expected to increase to $\$ 13$ billion by FY27 [10].
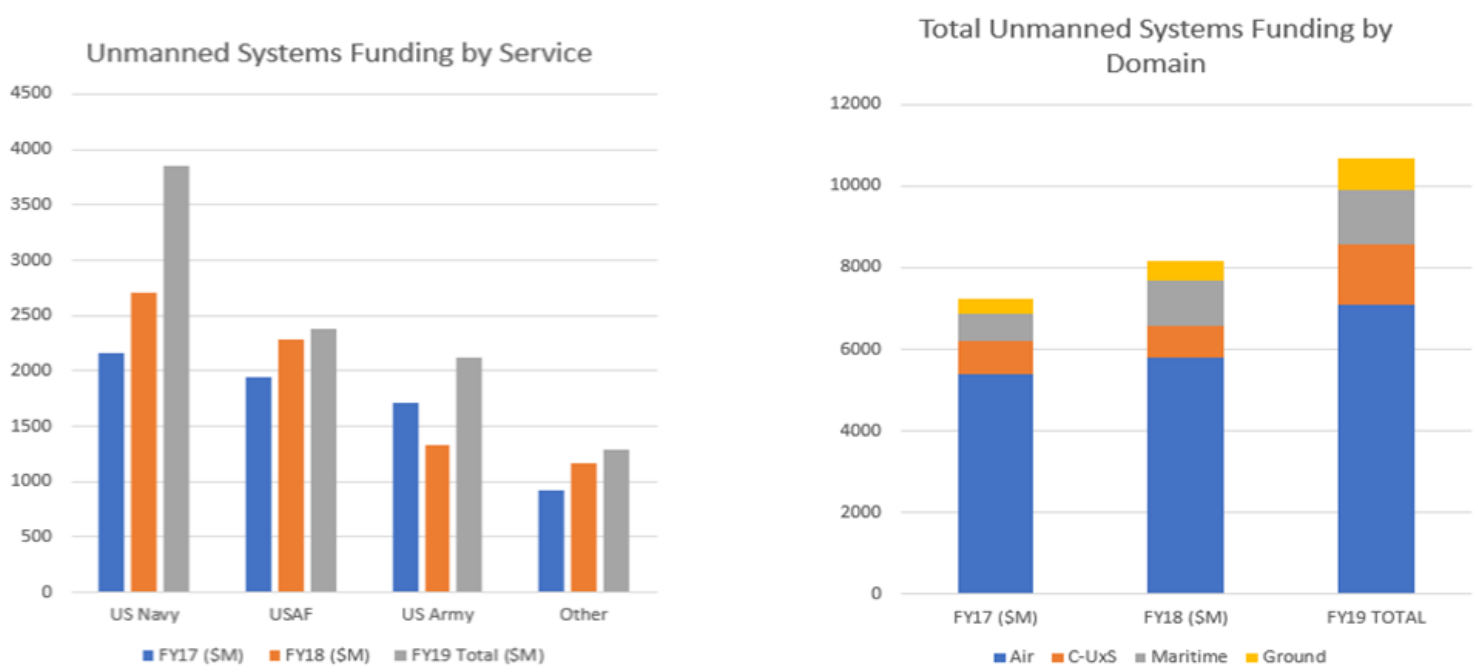

Figure 1. Estimated FY2019 DoD budget for unmanned systems acquisitions and RDT\&E [10].

Even though funding for UAS research and development is expected to expand, acquisition managers are still forced to make time and resource decisions regarding which UAS capabilities to develop. The money pool, while vast, remains finite and not all avenues of production are feasible. It would be very helpful if system developers could focus their efforts on the most realistic applications for future use. This research attempts to provide that realism by predicting which future UAS applications are most likely to be realized, the expected levels of autonomy permitted per application, and the primary challenges toward implementation. The study will do so by understanding the perspectives of current UAS stakeholders working in program offices, academia, and the pilot communities. Their view of future autonomous UAS capabilities and potential uses may help planners and developers anticipate where developmental investments should be made, where systemic barriers may exist, and how to better realize the benefits of autonomy in UAS. Ultimately, the ability to better understand the direction autonomous UAS capabilities are likely to take may allow planners to better translate user inputs into system requirements while also helping to reduce technical and safety risks. The earlier this is done, the stronger foundation future programs will have and the more cost-effective UAS systems will be. This study helps provide such focus. First, however, is a need to understand the role of autonomy within UAS systems.

\subsection{Autonomous Unmanned Aerial Systems (UAS)}

With respect to UAS, autonomous capabilities encompass the technologies that enable unmanned flight and autonomous behavior in the absence of an onboard pilot. However, when discussing whether a system should be considered autonomous, it is more accurate to discuss autonomy with respect to specific tasks or behaviors. Autonomy then can be considered as a capability (or a set of capabilities) that enables actions of a system to be automatic or within programed boundaries, or 
"self-governing" [10]. That is to say, an autonomous UAS may have autonomous landing capabilities, or may be able to autonomously perform air-to-air refueling, or may be able to coordinate among other UAS in a swarm construct to determine which UAS is best suited to perform a particular task. It is important to note that when a system is described as autonomous, it is not described as being fully autonomous with respect to all tasks. Rather the definition of autonomy applies only to a specified capability a UAS possesses.

Currently, all autonomous systems are supervised by human operators at some level with limited software-enabled capabilities, actions, or decisions delegated to the system. Conventionally, autonomy levels are inversely proportional to the degree in which human interaction is necessary; this degree is often described in terms of human independence (HI) [11].

The three categories of autonomy described for UAS operations within the HI context are: semi-autonomous, human-supervised, and autonomous. The semi-autonomous system is one that "once activated, is intended to only engage individual targets or specific target groups that have been selected by a human operator" [12]. This semi-autonomous description is what is often termed human in the loop (HITL); where the machine performs a function for a period of time, then waits for human input before continuing. The human-supervised, or human on the loop (HOTL) construct involves machines that can perform a function on their own, but have a human in a supervisory role, who can intervene if the machine fails or malfunctions. The human-supervised autonomous system is "designed to provide human operators with the ability to intervene and terminate engagements." When the machine can perform a function on its own and humans are unable to intervene, the category of autonomous system is referred to as autonomous, or human out of the loop (HOOTL) and is considered fully autonomous [12].

The potential range of applications for UAS is seemingly limitless and there is little doubt technology will advance to where HOOTL operations are possible. Within military operations, capabilities such as cargo or supply delivery and air-to-air refueling are being considered from a HOOTL level of autonomy. Realistically, however, some capabilities have a greater likelihood of success based on factors ranging from technical complexity to political acceptability. For example, there is widespread public concern and uneasiness about "silent-killer" robots roaming the skies or even fear of privacy invasion that have led to the adoption of laws that highly curtail local UAS operations $[13,14]$. These legal/political considerations are as important to overcome as the technical challenges. This study provides a user-based projection of which technologies are most likely to be realized over the next 20 years as well as any challenges each capability faces for actual implementation.

\subsection{UAS Defintiion and Functionalities}

Unmanned aircraft have different connotations to different groups. For this study, UAS is a "system whose components include the necessary equipment, network, and personnel to control an unmanned aircraft" [12]. This definition which was supplied to study participants, while broad, encompasses the wide array of military terms for unmanned aircraft, including: unmanned aerial vehicles (UAV), drones, and remotely piloted aircraft (RPA) $[7,12,15,16]$.

Further, UAS were not assumed to have any pre-determined limits regarding their functional capabilities beyond the basic elements which enable the aircraft to navigate, communicate, and accomplish controlled flight much the same as a manned aircraft, but with a suite of software and sensors that allow the UAS to operate without an onboard pilot. Embedded logic and rules typically include safety, system health monitoring, and contingency functionality were assumed to be existing functions of a working UAS. Instead, panelists participating in the study were expected to identify various mission capabilities UAS are capable of supporting. Such functions may include, but were not limited to, command and control (C2), intelligence collection/processing, offensive or defensive military movement and maneuver, and sustainment operations. Given the military background of study participants, functionalities were assumed to be understood by all panelists. 


\section{Materials and Methods}

This study employed the Delphi method which structures and facilitates discussion among groups of experts on complex topics through iterative rounds of questions with the goal of arriving at a consensus about a particular outcome [17]. The method is commonly used in policy development, technology forecasting, medical and education planning. It is primarily used as a planning or forecasting tool and is most applicable to deal with uncertainty in an area of imperfect knowledge. As there are no "correct" or verifiable answers, a consensus is considered an acceptable second choice [18]. Experts are carefully selected based on specific criteria related to the field of interest so that a broad spectrum of opinion on the topic can be examined. The group typically involves competing stakeholders so that conflicting demands can be considered by other group members. With the guidance of a facilitator (i.e., moderator or researcher), the group ultimately makes predictions about some future direction as it relates to the area of discussion. Figure 2 depicts the general process a Delphi method employs.

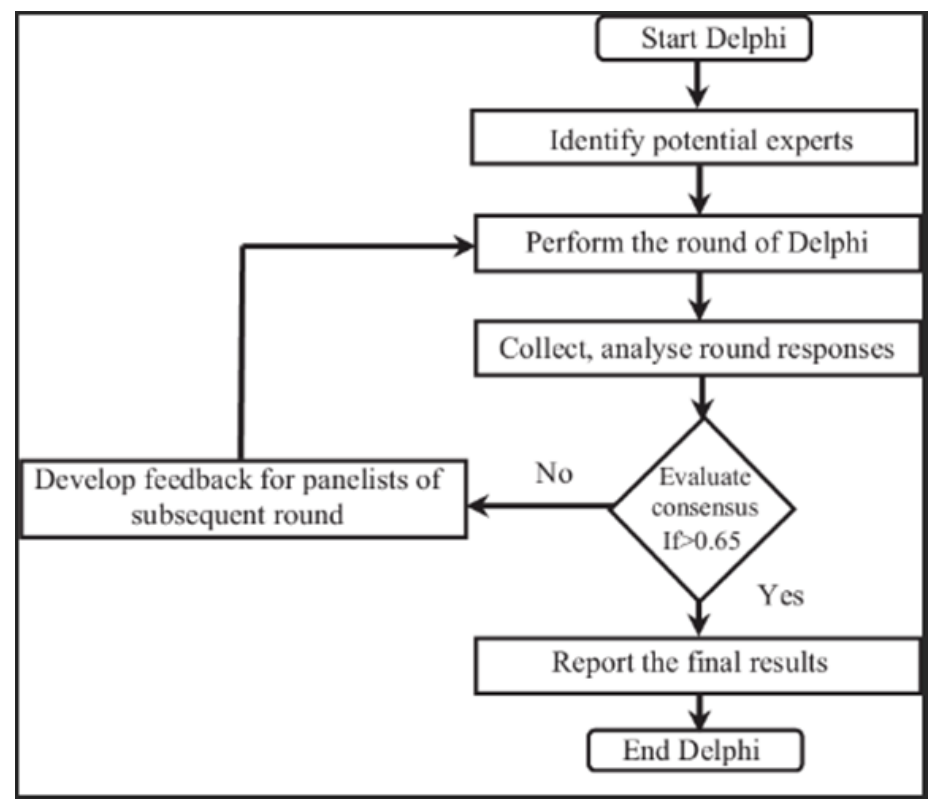

Figure 2. General example of the Delphi method.

The origins of the Delphi method come from a 1950s Air Force-sponsored RAND Corporation defense research project titled "Project Delphi" whose purpose was to aid in policy formulation and to forecast the impact of technology on warfare [17]. Since then, the Delphi method has been applied by government agencies, military services, and industry to identify areas for future research [19]. Even fields such as engineering, economics, and medicine have also benefitted from the application of Delphi studies $[19,20]$.

For this study, the consensus criterion was set at $70 \%$ or higher. Others using the Delphi method have used consensus levels as low as $60 \%$ [20], while other authors recommend a much higher value of $80 \%$ [21]. Using a lower consensus value can result in a larger number of recommendations in the results, but this can affect validity of the results. Selecting $70 \%$ was determined to be a good middle value of those found in in the literature.

This study uses the Delphi method to focus on four aspects of the UAS domain space including: (1) identification of current UAS usage, (2) prediction of future mission usage, (3) evaluation of current and future autonomy levels, and (4) perceived challenges to achieving future autonomous mission capabilities. As all panelists possess a military background, the questions were scoped to capture military specific missions. Many civilian UAS applications exist outside of the military; however, this study was limited to consideration of current and future military UAS operations. 


\subsection{Research Objectives}

Research objectives were designed to help UAS developers and researchers identify which mission areas may be most suitable for future autonomous UAS applications and what challenges may be encountered to reaching those missions. These objectives also served as the foundation for which drove the research instrument panelists used to respond to the Delphi study. In general, the objectives were as follows:

1. Determine what military missions are currently assigned to UAS and what level of autonomy is currently used for each mission.

2. Identify which UAS missions are most likely in 20 years and what level of autonomy future missions are likely to possess.

3. Identify challenges to implementation for future missions.

\subsection{Participant Selection}

For this study, in order to ascertain whether various stakeholder had similar or disparate views about current and future autonomous UAS missions, it was desirable to have multiple panelists representing pilots, acquisitions, and academia. Five pilots were included in the study, however, two were lost to attrition after receiving the first research instrument. Similarly, acquisition professionals from various program offices and specialty backgrounds were contacted for potential participation. Initially, five participants from acquisition backgrounds were scheduled to participate, however, two would eventually drop out prior to sending out the first research instrument. Two academia members currently conducting research into UAS technologies and with direct experience teaching UAS technology were also available to participate. The resulting panelists and their relevant experience are depicted in Table 1.

Table 1. Panelist selection criteria.

\begin{tabular}{ccccccccc}
\hline & \multicolumn{9}{c}{ Panelist Identification Number (PID\#) } & & \\
& PID01 & PID02 & PID03 & PID04 & PID05 & PID06 & PID07 & PID08 \\
\hline Experience Type & & Pilot & & \multicolumn{2}{c}{ Acquisitions } & Academic \\
\hline Civilian & & & & $\mathrm{x}$ & $\mathrm{x}$ & $\mathrm{x}$ & $\mathrm{x}$ \\
\hline Mil Exp & $\mathrm{x}$ & $\mathrm{x}$ & $\mathrm{x}$ & $\mathrm{x}$ & & $\mathrm{x}$ & $\mathrm{x}$ & \\
\hline Flight Exp & $\mathrm{x}$ & $\mathrm{x}$ & $\mathrm{x}$ & $\mathrm{x}$ & & & $\mathrm{x}$ & $\mathrm{x}$ \\
\hline Years of UAS Exp & 12 & 7 & 6 & 12 & 4 & 4 & 10 & 8 \\
\hline
\end{tabular}

The minimum criteria for participating as a panelist in the study was at least four years of experience in a UAS related field of work. Each participant interacted with the study via email.

\subsection{Research Instrument}

Construction of questions for the Delphi study began with careful consideration of the format. A common guide for describing autonomy was placed at the beginning of each research instrument sent to participants. For this study autonomy was defined as depicted in Figure 3.

In round one, panelists were tasked to identify current UAS mission areas and the level of autonomy employed for each mission on a 5-point Likert scale based on Figure 3. They were encouraged to provide rationale for their answers and as much detail as they wished. The questions were open-ended to allow respondents to answer in their own words as well as ask for clarification on areas not understood. Panelists were also asked to identify which military missions UAS were likely to become operational over the next 20 years, the level of autonomy expected to be employed for those missions, and the top two challenges to be addressed to ensure successful fielding of those missions. Round two had a more structured approach with aggregated information from round one reintroduced to ascertain consensus over previous inputs. 


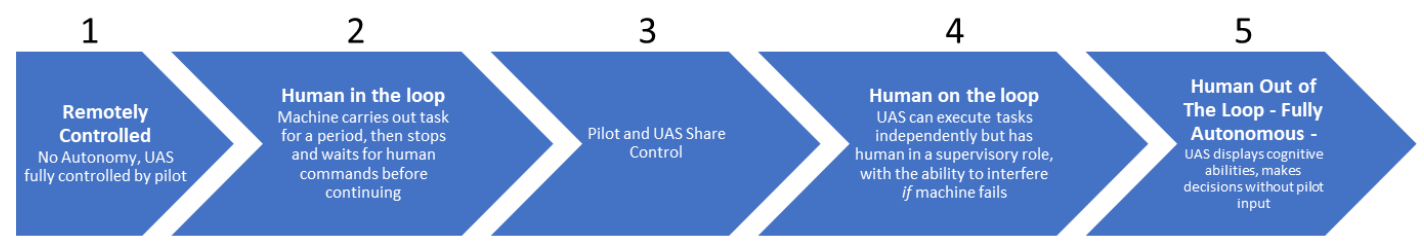

Figure 3. Likert scale used for level of autonomy assignment.

\section{Results}

Results are reported using a combination of a box-chart to show the mean and range of responses by panelist group and by calculating if consensus ( $>70 \%$ agreement) was achieved by all of the panelists $(n=8)$. Additionally, the overall response average and standard deviation are reported to further clarify the results depicted in the box-charts. It can be interpreted for a given a 5-point Likert scale, standard deviation (SD) $>1$ is unacceptably large and the results should be considered questionable and for this study will be discarded.

Round one served the dual purpose of establishing a common starting point to assess how panelists viewed current UAS missions and their levels of autonomy as well as creating lists of possible future missions, expected levels of autonomy, and associated challenges. Each panelist was asked to list current UAS missions they were aware of and assign a level of autonomy to the extent that they believed current missions employed autonomy using a 5-point Likert scale. Figure 4 shows the four mission areas the panelists identified and levels of autonomy each group assigned to each mission.

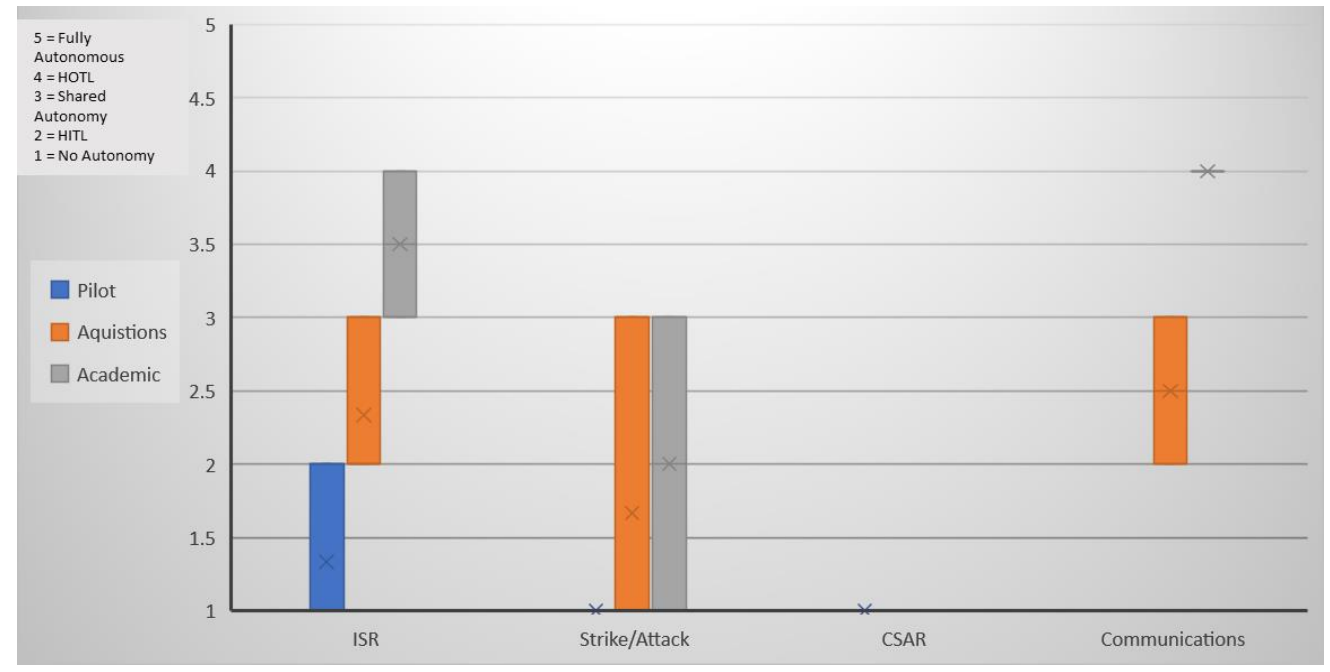

Figure 4. Current missions and assigned autonomy levels.

Intelligence, surveillance, and reconnaissance (ISR) and strike/attack were the two military missions identified by all panelists. Communications and combat search and rescue (CSAR) were also identified, but not by all panelists. Interestingly the pilot subgroup assigned the lowest levels of autonomy for any of the missions. Analysis of panelist comments revealed acquisition and academic panelists viewed the question as one of UAS capability, whereas pilots reported how autonomously the UAS were being used regardless of capability. Pilots cited reasons such as trust and mission complexity as reasons greater autonomy was not employed. These initial results signaled a potential disparity between what developers and academics believed could happen versus operational realities in the field.

In the second round, panelists were asked to agree or disagree with the predicted future UAS mission areas and rate the potential challenges importance to solve and likelihood of being solved within the next 20 years. The following sections highlight those results. 


\subsection{Future Missions and Assignment of Autonomy Levels}

All panelists were tasked to predict the likelihood each future mission would become operationalized over the next 20 years. They assigned a likelihood rating based on a 5-point Likert scale. Figure 5 reports the results of the panelists' responses.

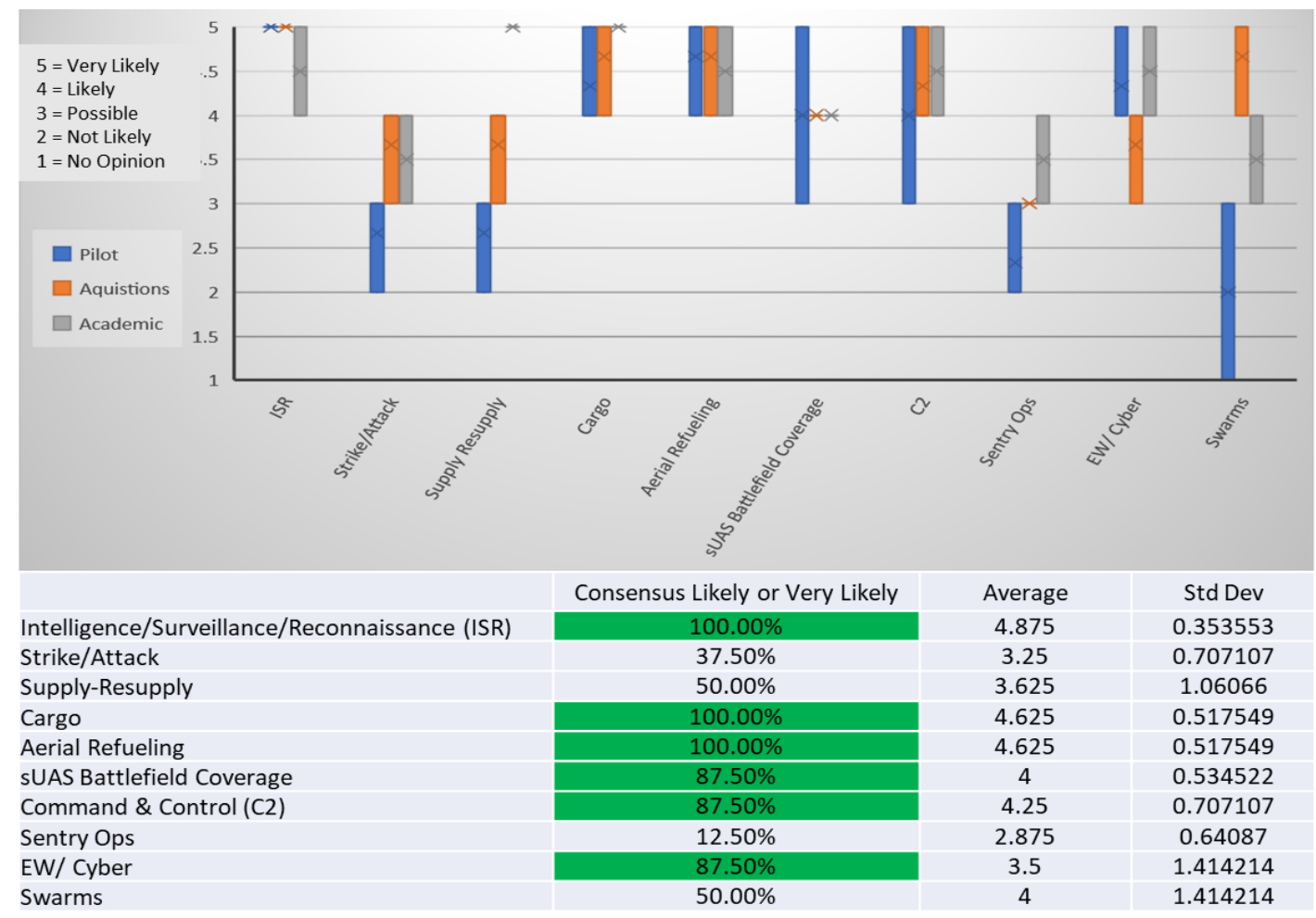

Figure 5. Assessment of future mission likelihood.

From Figure 5, ISR, cargo and aerial refueling appear to have the achieved consensus for expected development over the next 20 years. However, examining the average rating, panelists agreed 6 of the 10 predicted mission areas were likely or very likely to be operationalized over the coming 20 years. As mentioned previously, consensus was considered achieved when $70 \%$ or more of the panelists agreed. It should also be noted that of the 6 mission areas considered likely, electronic warfare (EW)/cyber had a large standard deviation (1.414) which indicates a large disparity between panelist responses although consensus did reach $87.5 \%$. As a result, caution is urged when including EW/cyber as a probable future mission.

Interestingly, not all the panelists agreed the original 10 mission areas are likely to become operational in the military. This insight may provide UAS developers and researchers direction for the most fertile areas for development. As noted earlier, research and development funds are finite. Pursuing the most likely options to succeed would be a wise choice.

In addition to projecting mission likelihood, panelists were also asked to assess the level of autonomy each mission area is expected to employ. Figure 6 depicts panelists predicted levels of autonomy by mission area. Pilots remained the most conservative toward the assignment of autonomy levels across all mission types with an average rating of 2.53 (between HITL and shared autonomy). Acquisition and academic panelists, however, believed future UAS missions will achieve autonomy levels between HOTL and fully autonomous (mean $=4.27$ and 4.2 respectively). While the differences between pilots and acquisition/academics is interesting to note, some consensus exists on expected levels of autonomy. ISR and swarms did achieve consensus at 70\% agreement for HOTL or higher levels of autonomy. While differences between pilots and other panelists, it should be noted pilots 
were consistent with other groups in shifting toward greater utilization of autonomous capabilities for future missions.

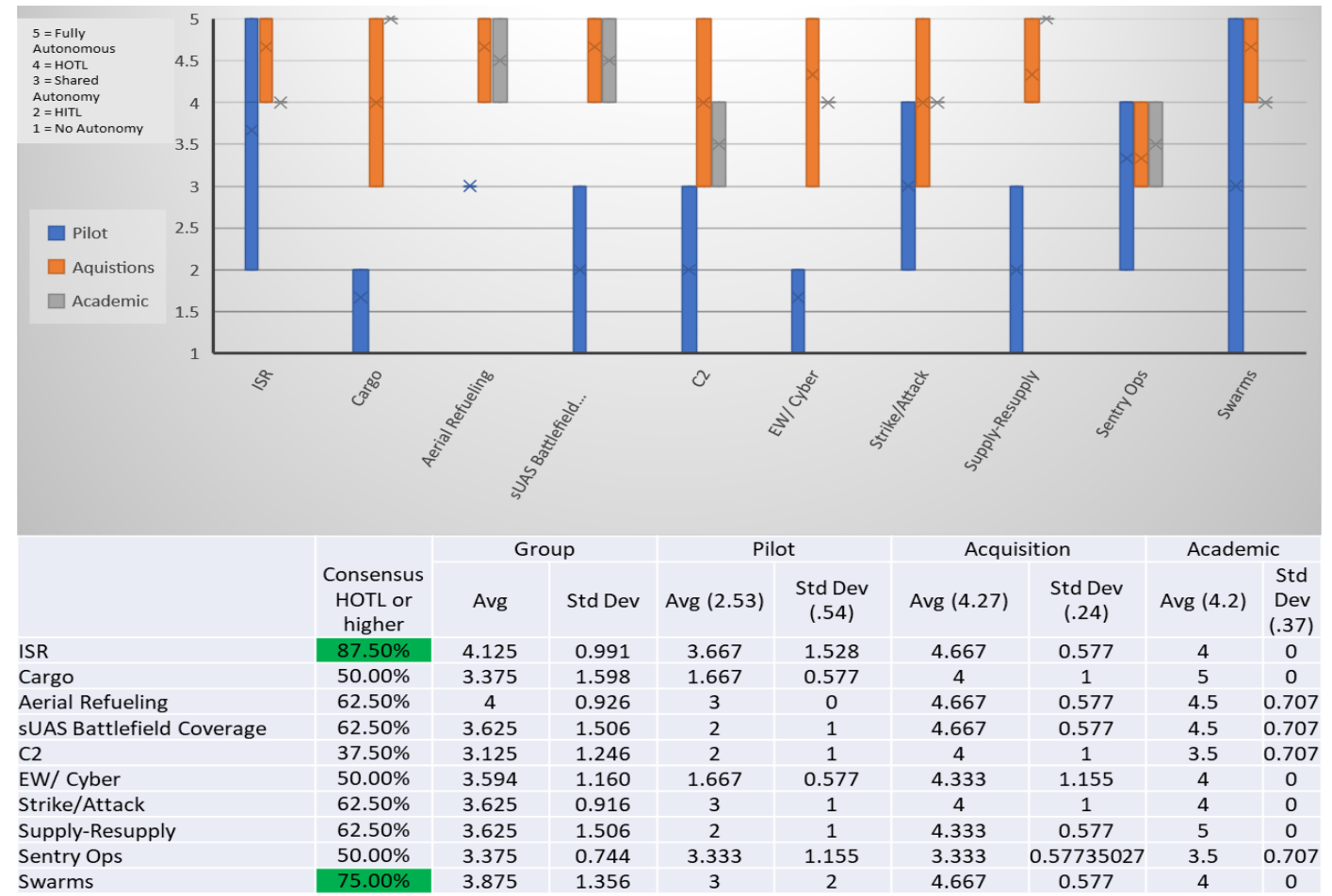

Figure 6. Future missions predicted autonomy levels.

Interestingly, if a lower consensus level (60\%) had been used, as recommended by Pham et al. [20], 6 of the 10 mission areas are then predicted to see autonomy levels of HOTL or higher. For this study, however, any results and recommendations will remain based on the $70 \%$ threshold discussed previously. Therefore, when combined with the with the expected likelihood results, program managers and researchers interested in maximizing research and development funding would perhaps find the greatest results pursuing autonomy in ISR applications, given swarms were not seen as particularly likely to be realized over the next 20 years by the panelists.

\subsection{Challenges to Future Use of UAS Autonomous Capabilities}

After panelists predicted the likelihood future missions would become operational, they were then asked to evaluate the previously identified challenges to implementing mission capabilities autonomously. Identifying the challenges is the first step toward developing strategies to address obstacles and achieve more advanced autonomous UAS capabilities. The challenges were naturally categorized into three groups: technological issues, data requirements, and programmatic issues. Each of these areas were further broken down into descriptive subcategories. Panelists were asked to also indicate the level of importance of each challenge subcategory and the likelihood the challenge can be solved within the next 20 years. The following sections discuss panelist responses, identifies trends, areas of interest for UAS developers and researchers.

\subsubsection{Technology Challenges}

Technology challenges facing future UAS autonomous mission capabilities include the eight subcategories depicted in Figure 7. Sense and avoid was considered by panelists as the ability of UAS to sense its environment and avoid any obstacles, including other UAS. Machine learning (ML)-artificial intelligence $(\mathrm{AI})$ recognized the increasing role of $\mathrm{AI} / \mathrm{ML}$ in the modern data analysis, especially 
under complexity and time constraints commonly found in aerial operations. Human-machine tech highlighted the panelist's belief that humans must be able to interact in a more natural way with UAS than traditional pilot interfaces such as keyboards, mice and joysticks. System complexity represents the technical problems associated with increasingly complex UAS design and operation. Matching evolving tech exemplified the panelist's belief that industry would develop cutting edge UAS technology faster than the military could and as such the military should harness that expertise. Data links referred to UAS having hardware and software required to establish data links with ground stations as well as other aircraft within the area of operations. Secure and reliable connectivity referred to the ability of the UAS to have secure and reliable communication channels back to the ground stations, especially over significant geographic distances.

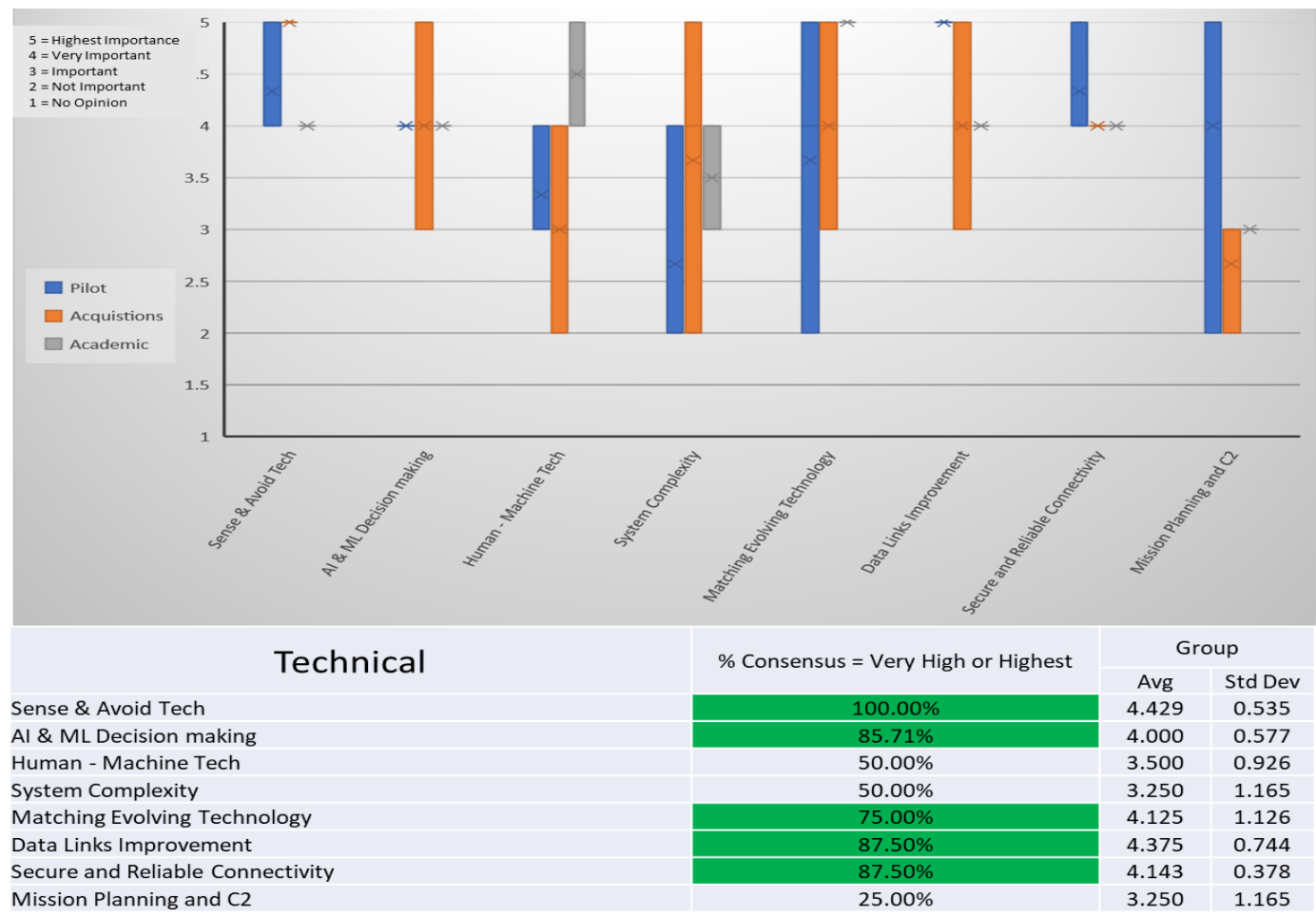

Figure 7. Technical challenges level of importance.

There was consensus that 5 of the 8 technology challenges were very important or higher to solve for the success of future autonomous UAS missions. Only human-machine tech, system complexity, and mission planning/C2 were not considered very important or higher by the panelists.

It was interesting to note panelist groups each viewed different Technical challenges as having the highest importance. The pilot group believed solving the data links technology challenge was of the "Highest Importance" (mean = 5.0, SD =0). In their comments, pilots relayed this challenge was a real-world problem today and anticipated it would have the largest impact on how much autonomy UAS would have in the future. The acquisition panelists listed sense and avoid technology as the 'Highest Importance' to future autonomous UAS capabilities (mean $=5.0, \mathrm{SD}=0$ ). They reasoned that at higher levels of autonomy, UAS would absolutely require answers to this challenge. Finally, academics believed the matching evolving tech (mean $=5.0, \mathrm{SD}=0$ ) was the top challenge in large part to their belief that commercial UAS development would rapidly out pace military research and development over the coming decades.

In addition to determining importance of challenges, panelists were asked to evaluate how likely it was each challenge would be overcome in the next 20 years. Figure 8 displays the likelihood rating assigned to the technical challenge. 


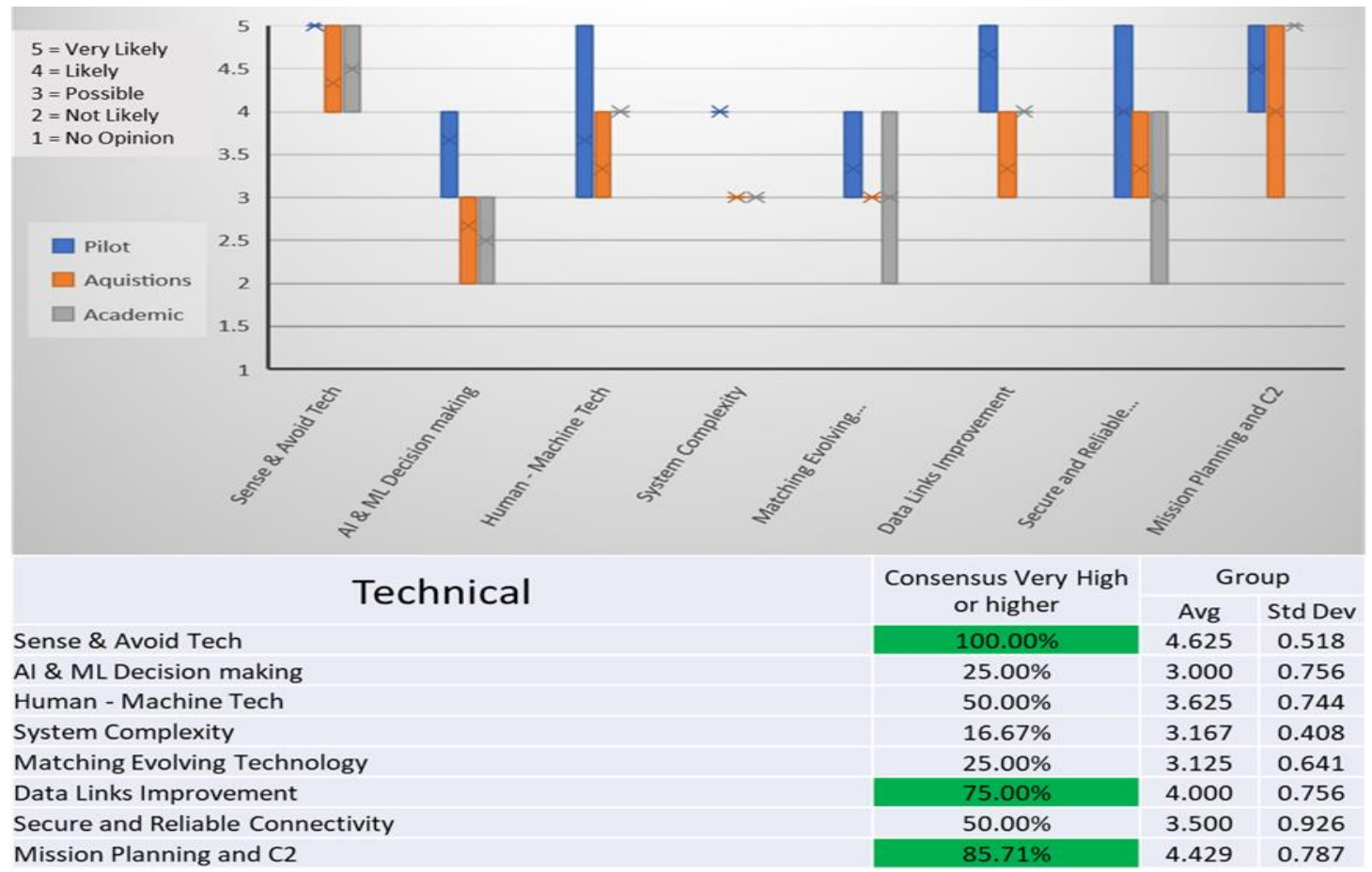

Figure 8. Likelihood that technical challenges will be solved.

Of the three challenges panelists agreed were most likely to be solved, only sense and avoid and data links improvement match the top challenges agreed upon by the panelists. These findings indicate UAS developers and researchers should focus efforts on developing solutions to improve UAS awareness and data link capabilities.

\subsubsection{Data Challenges}

In addition to technology challenges, panelists also identified data-related issues as a challenge to future autonomous UAS use. Figure 9 shows panelist assessed importance of eight data related challenges. Even though this was considered a challenge area separate from technology, themes such as sense and avoid and data links started to emerge.

Sense and avoid data referred to the ability to collect, process and store relevant data to avoid collisions while improved data links refers to the capacity to transfer and share large volumes of sensor and mission critical data. Integrate with Existing Systems reflected compatibility issues regarding the need to share data with existing military systems (on the ground and in the air) and integrate with civil and military acknowledged that UAS would need to share some data with civilian and military air traffic control (as well as other aircraft). Data volume software considered that many platforms in use today could not process, or in some cases, understand the data being sent. In a related way, middleware interoperability was a possible remedy to potentially process and transform data to meet various user needs. Finally, algorithms, methods, techniques references the "knowledge" of how to solve rapid, near real-time data analysis problems while information analysis captures the availability and capacity of hardware to actually implement the former.

Again, sense and avoid as well as improved data links were both rated as having very high or higher importance level, which indicates a consistent them when considered in conjunction with technical challenges. Consensus was also observed with integrating with existing systems and software development. Unfortunately, panelists were less descriptive of why they assigned the ratings for this section.

When asked to consider the likelihood of solving data challenges, Figure 10 shows panelists assessed sense, detect, and avoid, improved data links, and integrate with existing systems as likely or 
higher to be solved. It is interesting to note the three data challenges panelists considered most likely to be solved are also among the most important challenges to solve. UAS developers and researchers would be well served to consider focusing their resources primarily on those data challenge areas.

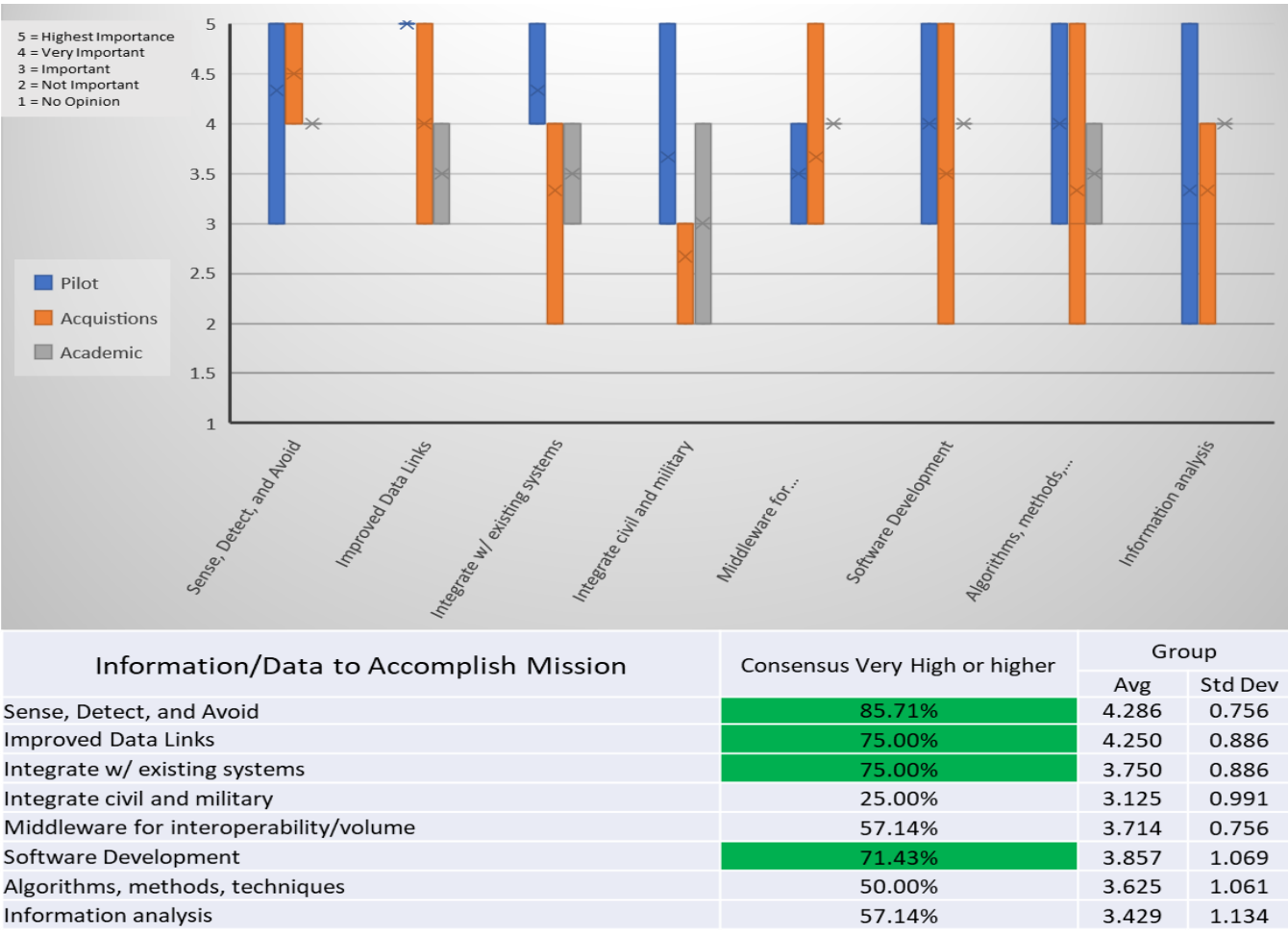

Figure 9. Data-related challenges' level of importance.

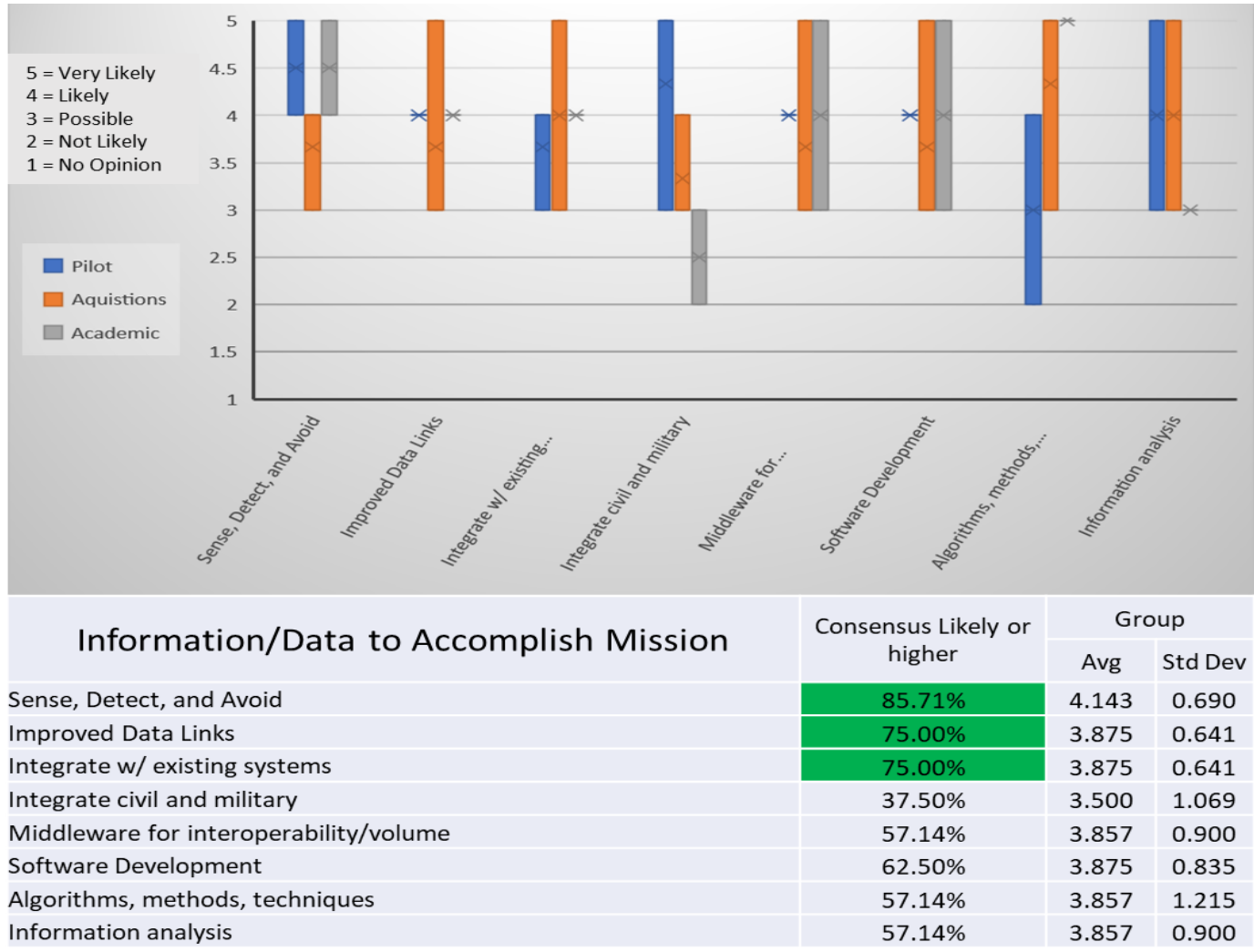

Figure 10. Likelihood that information/data challenges will be solved. 


\subsubsection{Programmatic Challenges}

The final challenge to future autonomous UAS operations was centered on programmatic issues. Figure 11 shows the challenges and level of importance as rated by the panelists.

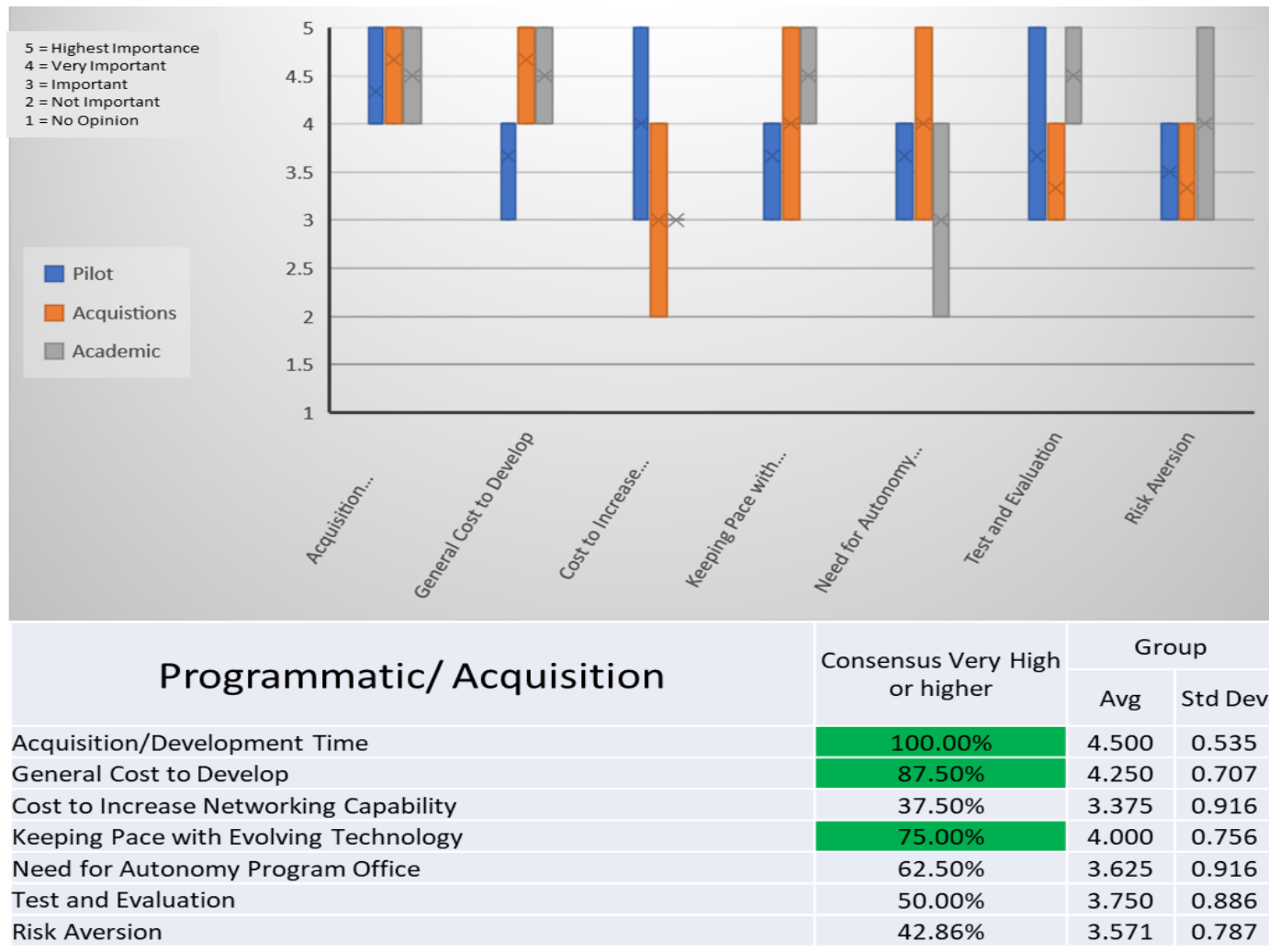

Figure 11. Programmatic-related challenges' level of importance.

Acquisitions and development time, general cost to develop and keeping pace with evolving technology were the three highest-rated programmatic challenge areas. They reflect the long lead times and high costs of developing new UAS platforms and the realization that technology often matures faster than the traditional military acquisition cycle. Panelists believed there could be risk of fielding UAS that were outdated before first deployment. General cost to develop new technology was very closely related to acquisition and development time, however the former emphasized time, while the latter focused on cost.

When asked about the likelihood to solve programmatic challenges, Figure 12 shows panelists were less optimistic the challenges would be solved in the next 20 years. There was consensus that cost to increase networking capability and risk aversion could be mitigated, but both had low means (3.750, $3.429)$ and high standard deviations $(1.165,0.976)$ which indicates weak consensus and wide variation between panelist responses. Comments from multiple panelists seem to indicate a general sense that programmatic issues were "too large" or "too complex" to be solved within a 20-year timeframe or even possibly ever. UAS developers and researchers could still use the most important programmatic challenge areas to focus their work; however, the only one deemed likely to be solved was focusing on driving down costs to increase network capability. 


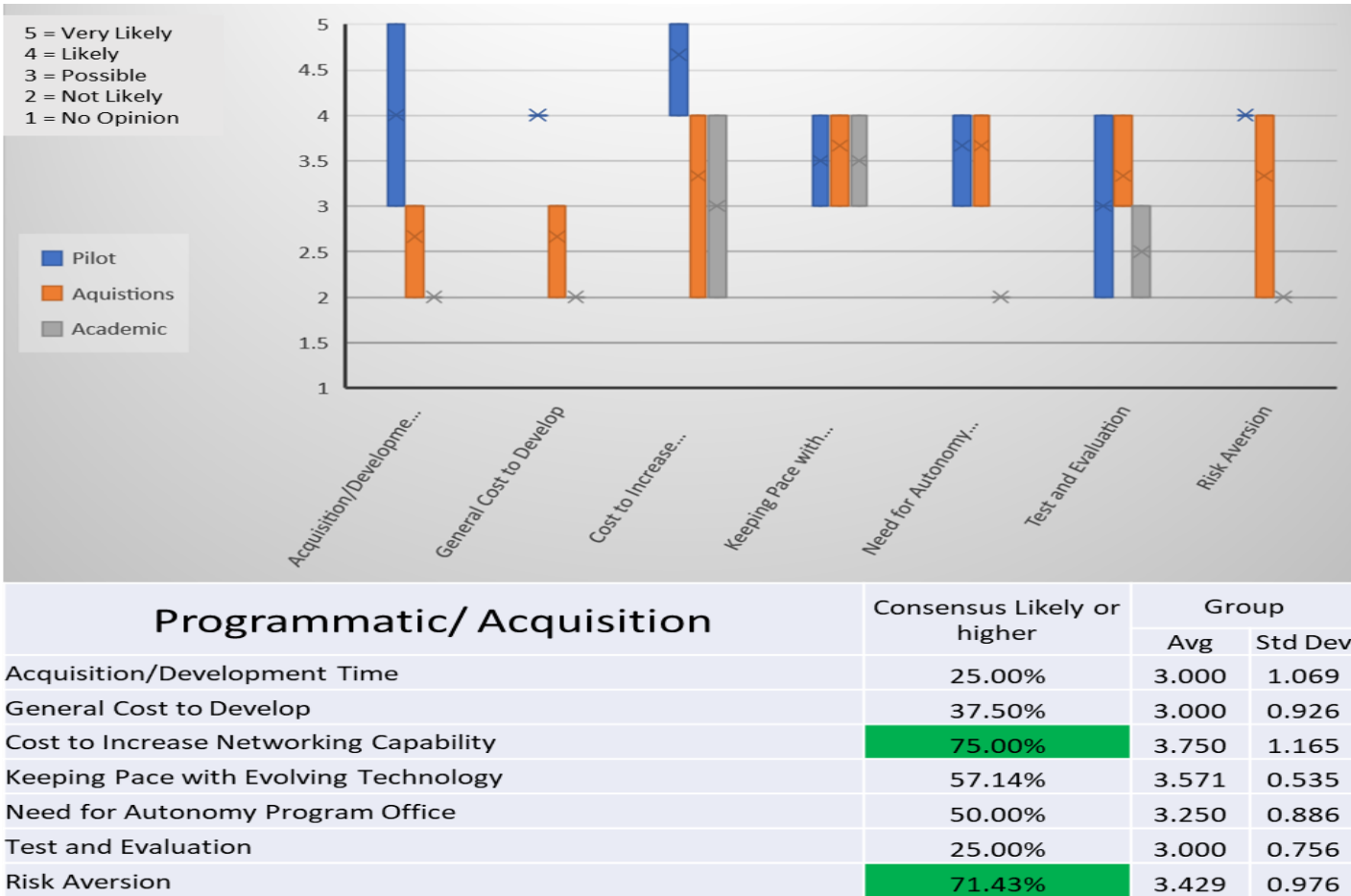

Figure 12. Likelihood that programmatic/acquisition challenges will be solved.

\section{Discussion}

The two-round Delphi study captured insights from pilot, acquisition, and academic communities. Overall, panelists predict an increased number of UAS mission areas over the next 20 years with a corresponding increasing level of autonomy for each mission area. This prediction is not only consistent with previous research, but also identifies specific mission areas in which the technology is likely to be applied. Specifically, ISR mission capabilities were identified as not only likely to expand over the next 20 years, but also to include the highest levels of autonomous UAS behavior, which is consistent with other studies on use of UAS technology [22-24]. Also, with regard to autonomy, the pilot group consistently predicted the lowest levels of future autonomy, which may indicate operators believe that while UAS may be capable of performing missions autonomously, external factors limit how autonomous the UAS systems may become. Follow-on studies may benefit from exploring differences between pilots and other panelists more rigorously by using methods promoted in recent studies in the data-driven decision-making (DDDM) field such as the similarity confirmation method described by Zhuang et al. [25].

In addition to identifying UAS mission areas, panelists revealed challenges that must be overcome in order to realize the potential of the projected mission capabilities. In general, the panelists agreed upon three challenge areas (technology, data, and programmatic); however, variation existed between panelist groups in terms of which challenges were most important. Interestingly, the top challenges, regardless of category, revolved around sensor technology, the data generated by the sensors, and the ability to process and share the data. These findings are consistent with related research into the top challenges facing future UAS use [26-28]. UAS researchers and developers would do well consider assigning the highest priorities to addressing these challenges, specifically as they relate to the 10 mission capabilities predicted by the study.

\section{Conclusions}

Although spending on UAS technology has continued to increase year-on-year, specific decisions must still be made about where to focus funding and efforts. Leveraging the knowledge of which 
mission areas are most likely to be realized and addressing the challenges reported, this study can be used as inputs for decision makers to help determine where to dedicate resources to maximize the advancement of autonomous UAS capabilities. Such a task is essential as it is clear that increasingly autonomous UAS capabilities are evolving rapidly and will emerge as indispensable tools.

Author Contributions: Conceptualization, A.S. and B.L.; methodology, A.S.; validation, A.S. and B.L.; formal analysis, A.S. and B.L.; investigation, A.S.; data curation, A.S.; writing-original draft preparation, A.S.; writing-review and editing, B.L.; supervision, B.L.; project administration, B.L.; funding acquisition, B.L. All authors have read and agreed to the published version of the manuscript.

Funding: This research was funded by a grant from the Air Force Office of Scientific Research to explore Dynamic Data Driven Applications, Erik Blasch, Principle Investigator, grant number 18RT0095.

Conflicts of Interest: The authors declare no conflict of interest.

\section{References}

1. Newcome, L.R. Unmanned Aviation: A Brief History of Unmanned Aerial Vehicles; American Institute of Aeronautics and Astronautics, Inc.: Reston, VA, USA, 2004.

2. Watts, A.C.; Ambrosia, V.G.; Hinkley, E.A. Unmanned Aircraft Systems in Remote Sensing and Scientific Research: Classification and Considerations of Use. Remote Sens. 2012, 4, 1671-1692. [CrossRef]

3. Ditzler, G.; Hariri, S.; Akoglu, A. High Performance Machine Learning (HPML) Framework to Support DDDAS Decision Support Systems: Design Overview. In Proceedings of the 2017 IEEE 2nd International Workshops on Foundations and Applications of Self* Systems, FAS*W, Tucson, AZ, USA, 18-22 September 2017; pp. 360-362. [CrossRef]

4. Straub, J. Consideration of the use of autonomous, non-recallable unmanned vehicles and programs as a deterrent or threat by state actors and others. Technol. Soc. 2016, 44, 39-47. [CrossRef]

5. Van Hien, N.; Van He, N.; Diem, P.G. A model-driven implementation to realize controllers for Autonomous Underwater Vehicles. Appl. Ocean Res. 2018, 78, 307-319. [CrossRef]

6. Zema, N.R.; Natalizio, E.; Ruggeri, G.; Poss, M.; Molinaro, A. MeDrone: On the use of a medical drone to heal a sensor network infected by a malicious epidemic. Ad Hoc Netw. 2016, 50, 115-127. [CrossRef]

7. Norton, T.L.; Col, L. Staffing for Unmanned Aircraft Systems (UAS) Operations; IDA Paper P-5253 Log: H 15-000499; Institute for Defense Analysis: Alexandria, VA, USA, 2016.

8. Who Has What: Countries with Drones Used in Combat. 2018. Available online: https://www.newamerica. org/in-depth/world-of-drones/2-who-has-what-countries-drones-used-combat/ (accessed on 14 February 2019).

9. Finnegan, P. Teal Group Predicts Worldwide Civil Drone Production Will Almost Triple Over the Next Decade. 2019. Available online: https://www.tealgroup.com/index.php/pages/press-releases/60-teal-grouppredicts-worldwide-civil-drone-production-will-almost-triple-over-the-next-decade (accessed on 3 March 2020).

10. Defense Science Board. Task Force Report: The Role of Autonomy in DoD Systems. Task Force Repoer. July 2012. Available online: https://fas.org/irp/agency/dod/dsb/autonomy.pdf (accessed on 3 March 2020).

11. Huang, H.-M. Autonomy levels for unmanned systems (ALFUS) framework. In Proceedings of the 2007 Workshop on Performance Metrics for Intelligent Systems-PerMIS, Gaithersburg, MD, USA, 28-30 August 2007.

12. Williams, A.P.; Scharre, P.D. Autonomous Systems: Issues for Defence Policymakers; NATO Communications and Information Agency: Hague, The Netherlands, 2015.

13. Freeman, P.K.; Freeland, R.S. Politics \& technology: U.S. polices restricting unmanned aerial systems in agriculture. Food Policy 2014, 49, 302-311.

14. Dolan, A.; Thompson, R. Integration of Drones into Domestic Airspace: Selected Legal Issues; Congressional Research Office: Washington, DC, USA, 2013.

15. Cooke, N.J.; Rowe, L.J.; Bennett, W.; Joralmon, D.Q. Remotely Piloted Aircraft Systems: A Human Systems Integration Perspective; John Wiley and Sons Ltd.: West Sussex, UK, 2016.

16. Gupta, S.G.; Ghonge, M.M.; Jawandhiya, P.M. Review of Unmanned Aircraft System. Int. J. Adv. Res. Comput. Eng. Technol. 2013, 2. [CrossRef] 
17. Linstone, H.A.; Turoff, M. The Delphi Method-Techniques and Applications; Addison-Wesley: Reading, MA, USA, 1975.

18. Oliver, R.C.; Balko, B.; Seraphin, A.; Calhoun, A. Survey of Long-Term Technology Forecasting Methodologies; Document D-2430 Log: H 00-000164, December 1, 2002; Institute for Defense Analysis: Alexandria, VA, USA, 2002.

19. Mitchell, V.W. The Delphi Technique: An Exposition and Application. Technol. Anal. Strateg. Manag. 1991, 3, 333-358. [CrossRef]

20. Pham, T.; Gossec, L.; Fautrel, B.; Combe, B.; Flipo, R.M.; Goupille, P.; Le Loët, X.; Mariette, X.; Puéchal, X.; Wendling, D.; et al. Physical examination and laboratory tests in the management of patients with rheumatoid arthritis: Development of recommendations for clinical practice based on published evidence and expert opinion. Jt. Bone Spine 2005, 72, 222-228. [CrossRef] [PubMed]

21. Kelly, C.M.; Jorm, A.F.; Kitchener, B.A. Development of mental health first aid guidelines for panic attacks: A Delphi study. BMC Psychiatry 2009, 9, 49. [CrossRef] [PubMed]

22. Roma, A. Drones and popularisation of space. Space Policy 2017, 41, 65-67. [CrossRef]

23. Shishkov, B.; Hristozov, S.; Janssen, M.; van den Hoven, J. Drones in Land Border Missions: Benefits and Accountability Concerns. In Proceedings of the 6th International Conference on Telecommunications and Remote Sensing, Delft, The Netherlands, 6-7 November 2017; pp. 77-86.

24. Brumfield, E. Armed Drones for Law Enforcement: Why It Might Be Time to Re-Examine the Current Use of Force Standard. McGeorge Law Rev. 2014, 46, 543.

25. Zhuang, Z.Y.; Su, C.R.; Chang, S.C. The effectiveness of IF-MADM (intuitionistic-fuzzy multi-attribute decision-making) for group decisions: Methods and an empirical assessment for the selection of a senior centre. Technol. Economic Dev. Econ. 2019, 25, 322-364. [CrossRef]

26. Khoufi, I.; Laouiti, A.; Adjih, C. A Survey of Recent Extended Variants of the Traveling Salesman and Vehicle Routing Problems for Unmanned Aerial Vehicles. Drones 2019, 3, 66. [CrossRef]

27. Marx, A.; Chou, Y.; Mercy, K.; Windisch, R. A Lightweight, Robust Exploitation System for Temporal Stacks of UAS Data: Use Case for Forward-Deployed Military or Emergency Responders. Drones 2019, 3, 29. [CrossRef]

28. Sharma, V. Advances in Drone Communications, State-of-the-Art and Architectures. Drones 2019, 3, 21. [CrossRef]

(C) 2020 by the authors. Licensee MDPI, Basel, Switzerland. This article is an open access article distributed under the terms and conditions of the Creative Commons Attribution (CC BY) license (http://creativecommons.org/licenses/by/4.0/). 